\title{
Is the content and duration of the Graduated Return to Play protocol after concussion demanding enough? A challenge for Berlin 2016.
}

1. Simon Kemp ${ }^{1}$

2. Jon Patricios ${ }^{2,3,4}$

3. Martin Raftery ${ }^{5}$

Author Affiliations:

1. Chief Medical Officer, Rugby Football Union, London, UK

2. Sports Concussion South Africa, Johannesburg, South Africa

3. Faculty of Health Sciences, The Section of Sports Medicine, University of Pretoria, Pretoria, South Africa

4. Faculty of Health Sciences, The Department of Emergency Medicine, University of the Witwatersrand, Johannesburg, South Africa

5. Chief Medical Officer, World Rugby, Dublin, Ireland

Correspondence to:

Dr. Simon Kemp, Rugby Football Union, Twickenham Stadium, 200 Whitton Road, Twickenham, London TW2 7BA, UK E:simonkemp@rfu.com

\section{Introduction}

Determining the appropriate starting point and duration of the Return to Play (RTP) process after injury is fundamental to the practice of clinical sports medicine. The most commonly cited RTP framework after concussion is outlined as part of all international concussion consensus statements. This framework is a key element of concussion management across all levels of sport and provides a 'cornerstone' of current education initiatives. In Rugby Union, RTP guidelines last underwent a major update by World Rugby, the International Federation, in 2011 and are consistent with international concussion consensus.

There is a limited evidence base informing the content and duration of the Graduated Return to Play. Specifically, the evidence evaluating the effect, optimal amount and type of rest is sparse. Moreover, current return to play guidelines for children and adolescents are based on those of adults..

Whilst research concerning a range of clinical outcomes following concussive injury in sport continues to develop, until recently, little has been known about the consequences of return to play.

Increased injury risk following return to play

Three recently published studies in professional soccer players [1], American college athletes [2] and professional rugby players [3] have all identified an increased risk of injury following return to play from concussion. In addition, although not statistically significant, a further study highlighted a trend 
for concussed players to sustain more injuries upon return to play [4]. These increased relative risks of subsequent injury are noteworthy, particularly when contextualised against the pre-concussion risk of injury in professional rugby and soccer (Figure 1).

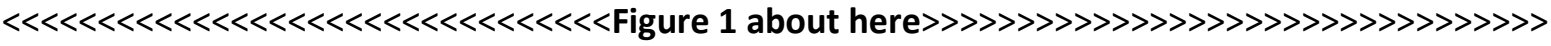

\section{Possible mechanisms for the increased risk of musculoskeletal injury after concussion}

The results from these studies raise a number of questions.

What is the underlying mechanism for this increase in susceptibility? Is it a consequence of the primary concussive injury whereby disruption of cortical pathways may result in slower reaction and movement times? Could impaired functioning of the vestibular system lead to reduced balance and proprioceptive function?

Alternatively, is the increased risk a consequence of the way recovery after concussion is currently assessed and managed? The current GRTP focuses on rest rather than on the re-development and re-training of neuro-muscular control, proprioception and co-ordination that is typically seen in the rehabilitation of musculoskeletal injuries.

Finally, it may be that the length of RTP is too short. Typically, the time to RTP after concussion is shorter than for the majority of other injuries. The average time taken for professional rugby players to return after concussion in the Cross study [3] is 11 days compared to an average of 21 days for musculoskeletal injury in the same cohort [5].

\section{Are the current tools used to assess recovery adequate?}

In light of this increased risk of injury following return to play, are the recommended assessment tools in the SCAT3 and the common computerised neuro-cognitive testing systems sufficiently sensitive and repeatable to detect subtle symptom, balance and cognitive deficits?

- Currently, assessments of recovery during the GRTP performed on an athlete at rest may not be valid predictors of in-game injury risk, and the benefit of post-exertional testing has been demonstrated [6].

- Neuromuscular control deficits have been observed experimentally, under dual-task conditions for example, abnormal gait may be seen with a cognitive distractor task [7].

- These deficits may be exacerbated during more demanding athletic activities, contributing to the increased rate of musculoskeletal injury following concussion.

- The benefits of more targeted rehabilitation focussing on cervical and vestibular interventions following concussion have also been demonstrated in studies [8].

Overall, we believe that there is a place for more physically and cognitively challenging athletic tasks as part of re-training and rehabilitation and as part of the assessment of recovery. Indeed, the introduction of a standardised exercise treadmill test into the RTP process has already shown promise in adolescents once normal tolerance to exercise had been established [9]. 


\section{Call to action - time to make the RTP protocol more challenging?}

We believe that the design and timescale of the GRTP protocol for professional athletes should be re-visited with greater emphasis on dual task activities, active re-training (cognition, balance \& vision) and the development of functional assessments performed during exertion in order to better meet the principles of other rehabilitation models. We suspect that the current GRTP may not be long enough or diverse enough in order to meet these aims.

The role of modifying factors in the prognosis of concussion has been acknowledged (if not quantified) and should influence RTP decision-making. More targeted clinical evaluation and interventions could further improve the specificity of the timing, duration and nature of the GRTP protocol.

The $5^{\text {th }}$ International Consensus Conference on Concussion in Sport in Berlin from October $26^{\text {th }}-27^{\text {th }}$, 2016 provides an opportunity to revisit GRTP guidelines for all sports. We post some considerations in the web appendix.

\section{References}

1. Nordström A, Nordström P, Ekstrand J. Sports-related concussion increases the risk of subsequent injury by about $50 \%$ in elite male football players. British journal of sports medicine 2014;48(19):1447-50

2. Lynall RC, Mauntel TC, Padua DA, Mihalik JP. Acute Lower Extremity Injury Rates Increase after Concussion in College Athletes. Medicine and science in sports and exercise 2015;47(12):2487-92

3. Cross MK, S; Smith, A; Trewartha, G; and Stokes, K. Professional Rugby Union players have a $60 \%$ greater risk of time loss injury after concussion: a 2-season prospective study of clinical outcomes. British Journal of Sports Medicine in press

4. Makdissi M, McCrory P, Ugoni A, Darby D, Brukner P. A prospective study of postconcussive outcomes after return to play in Australian football. The American journal of sports medicine 2009;37(5):877-83

5. Rugby Football Union. Premiership Rugby Injury Surveillance Project: 2013-14 Annual Report, 2015.

6. McGrath N, Dinn WM, Collins MW, Lovell MR, Elbin R, Kontos AP. Post-exertion neurocognitive test failure among student-athletes following concussion. Brain Injury 2013;27(1):103-13

7. Ingriselli JM, Register-Mihalik JK, Schmidt JD, Mihalik JP, Goerger BM, Guskiewicz KM. Outcomes, utility, and feasibility of single task and dual task intervention programs: preliminary implications for post-concussion rehabilitation. Journal of Science and Medicine in Sport 2014;17(6):580-85

8 Schneider KJ, Meeuwisse WH, Nettel-Aguirre A, et al. Cervicovestibular rehabilitation in sportrelated concussion: a randomised controlled trial. British journal of sports medicine 2014:bjsports-2013-093267

9. Darling SR, Leddy JJ, Baker JG, et al. Evaluation of the Zurich guidelines and exercise testing for return to play in adolescents following concussion. Clinical Journal of Sport Medicine 2014;24(2):128-33 